Voix et Images

voixetimages

\title{
Entretien avec Yves Préfontaine
}

\section{Pierre Ouellet}

Volume 24, numéro 1 (70), automne 1998

Yves Préfontaine

URI : https://id.erudit.org/iderudit/201404ar

DOI : https://doi.org/10.7202/201404ar

Aller au sommaire du numéro

\section{Éditeur(s)}

Université du Québec à Montréal

\section{ISSN}

0318-9201 (imprimé)

1705-933X (numérique)

Découvrir la revue

\section{Citer ce document}

Ouellet, P. (1998). Entretien avec Yves Préfontaine. Voix et Images, 24(1), 20-35. https://doi.org/10.7202/201404ar d'utilisation que vous pouvez consulter en ligne.

https://apropos.erudit.org/fr/usagers/politique-dutilisation/ 


\title{
Entretien avec Yves Préfontaine
}

\author{
Pierre Ouellet, Université du Québec à Montréal
}

V. et I. : Quels souvenirs gardez-vous des premiers textes que vous avez écrits?

Y. P. : J'ai commencé à écrire à l'âge de treize ans. C'était en 1950, plus précisément vers la fin de l'été, au phare de l'île Verte et à Trois-Pistoles, hauts lieux, à la fois mythiques et réels, de mon premier cheminement. Mais il me faut, d'abord, dire quelques mots du contexte dans lequel s'inscrit mon enfance. Du climat familial, notamment, qui n'était pas toujours drôle, tant s'en faut, mais qui recelait des traits plutột singuliers par rapport à l'environnement québécois moyen de l'époque. Avec le recul, je me rends compte du rôle que mon père, un homme à la fois terriblement ombrageux et généreux de son savoir, typiquement victorien par son éducation, a pu jouer dans ma "genèse".

Pour toutes sortes de raisons, qu'il serait trop long et sans doute inopportun d'expliquer ici, j'ai été plus ou moins séparé de ma famille pendant les dix premières années de ma vie. Je ne la voyais que les fins de semaine, à Montréal, où nous habitions, et pendant les vacances d'été à la grève de Trois-Pistoles, où nous avions un chalet, et à l'île Verte, dont le phare fut gardé par quatre générations de cousins liés à ma grand-mère paternelle, d'origine écossaise et irlandảise. Nous allions aussi assez régulièrement en Gaspésie, à Percé, aux Méchins ou à Grande-Rivière, alors principal port de pêche de la péninsule. On y voyait les barques encore à voiles des pêcheurs partir en mer tôt le matin et rentrer à la tombée du jour dans un flamboiement de soleil ou dans la pluie, la bruine et les tempêtes formidables qui faisaient aussi partie du quotidien. Ces lieux magiques et excessifs - la mer, les marées, les pierres splendides, les arbres de ce pays, "l'entière géologie", dirait Paul-Marie Lapointe - m'ont autant marqué que mes premières lectures d'auteurs essentiels.

V. et I. : La nature aura été votre premier "livre".

Y. P. : Le frère Marie-Victorin, maitre et ami de mon père, a joué un certain rôle dans l'orientation, après sa médecine, de sa carrière de chercheur en biologie marine, domaine où il a excellé une grande partie de sa vie à l'Institut de biologie de l'Université de Montréal; peut-être a-t-il aussi joué un rôle, indirectement, sur mon orientation poétique. Sous 
l'influence de l'auteur de La flore laurentienne (un grand livre de littérature, pas seulement de science), mon père lisait et m'apprenait à lire la nature comme on le fait d'un livre, en effet. Rien n'échappait à sa nomination des choses et des êtres: bêtes, plantes, arbres, pierres, jusqu'aux mousses et aux lichens qu'il nommait de leur nom latin et de leur nom courant. C'est par là, d'abord, que j'ai appris non seulement à lire mais sans doute aussi à "écrire" déjà, dans le souci d'une nomination juste et différenciée du monde de la nature.

V. et I.: La littérature ne pouvait venir, pour vous, qu'après la nature? L'expérience du monde ne pouvait que précéder celle de la parole?

Y. P. : Il y eut la musique, aussi, qui fut très tôt pour moi aussi vitale que l'eau. Particulièrement la musique qu'on qualifiait de moderne et qui l'est probablement moins aujourd'hui. J'y suis tombé, à la fin de l'enfance, comme Obélix dans la potion magique... Les premiers disques que j'ai achetés avec mon argent de poche, à quatorze ans, à New York, au cours d'un voyage avec mes parents, furent des enregistrements du Sacre du printemps (la Nature, encore!) et de Petroucbka de Stravinski. Un peu plus tard, j'ai découvert la seconde École de Vienne: Schœenberg, Berg, Webern. Je dévorais ces musiques comme des friandises empoisonnées qui nourrissaient mon angoisse et un tempérament exacerbé que je cultivais, bien sûr, avec une certaine complaisance...

V. et I. : Et les poisons littéraires? Votre père en avait sans doute à portée de la main dans son "laboratoire" ou sa "pharmacie"?

Y. P.: La maison croulait sous les livres et les revues. Évidemment, les sciences y dominaient, mais mon père était un scientifique humaniste, à l'ancienne, un "savant", comme on disait. Il éprouvait un immense intérêt pour la littérature. Ses goûts le portaient vers les grecs, les latins, les classiques français et anglais, mais sa bibliothèque était riche et diversifiée. Après y avoir découvert la poésie de Nelligan, dont la prosodie, à l'époque, me touchait plus ou moins, j'ai vécu mon premier choc littéraire en lisant Les îles de la nuit d'Alain Grandbois, dont mon père possédait un exemplaire dédicacé. J'ai déjà écrit il y a longtemps que le souffle, la cosmicité, l'ouverture au monde d'Alain Grandbois ont été pour moi des éléments déclencheurs déterminants. Ensuite vinrent les lectures de SaintDenys Garneau et d'Annè Hébert.

À la même époque, celle de mes treize ans, j'ai découvert, toujours dans la bibliothèque paternelle, un roman qui m'a profondément marqué: Matbieu de Françoise Loranger, dont Pierre de Grandpré et ses collaborateurs ont souligné, dans leur déjà ancienne Histoire de la littérature française $d u$ Québec, le sort injuste qui lui avait été réservé, sans le commenter ni l'analyser, toutefois, perpétuant ainsi le sort malheureux qu'il continue de subir... Je m'identifiais à ce misfit existentialiste des années quarante. Il va sans dire que certains passages érotiques, rarissimes à cette 
époque, troublaient le préadolescent que j'étais. Et puis il y eut un beau petit livre de poésie, Les équilibres illusoires, publié, je crois, aux Cabiers de la file indienne, par un ex-étudiant et ami de mon père, un ingénieur agronome qui signait du pseudonyme de Pierre-Yves Le Baron.

Je ne veux pas négliger, encore une fois, l'importance qu'a eue pour moi la lecture d'ouvrages de sciences naturelles, de botanique et de géologie surtout, où j'ai puisé des éléments de mon vocabulaire.

J'étais mûr, dès lors, pour la poésie. Et c'est ce qui advint cet été-là, à l'île Verte et à Trois-Pistoles. Je me mis à écrire comme un forcené et cela dura des années. Des poèmes, des poèmes scéniques injouables, porteurs du souffle énorme qui m'habitait et s'exprimait fort maladroitement, des poèmes chargés d'un érotisme morbide comme le vivent tant d'adolescents, surtout dans ces temps d'oppression de tout ce qui venait du Vivant et des Grands Rythmes.

V. et I. : Vous sentiez alors le besoin d'accorder vos rythmes poétiques à ceux de la nature?

Y. P. : Nous avions un petit télescope portatif à l'île Verte et lorsque nous allions ailleurs, en pleine forêt laurentienne, nous passions, avec mon père, des nuits entières à observer le ciel, à nommer les astres et les constellations, faisant ainsi entrer l'immense espace du cosmos dans l'espace étroit de la langue, qui s'élargissait, battant dès lors au rythme de la nature. D'une nature dont on devait décrypter les secrets, au-delà de la seule description.

V. et I.: Le lexique que votre poésie emprunte aux sciences naturelles rappelle un peu l'usage que Saint-John Perse fait du vocabulaire nautique et maritime non seulement pour sa précision technique mais pour ses qualités sonores, intrinsèquement poétiques.

Y. P.: Vous songez surtout à Amers. Oui, sans doute. Cette nomination, à la fois précise et imagée, est en fait une façon de s'approprier le sens du "paysage", que le grand poète anglais Gerard Manley Hopkins appelle inscape, "paysage intérieur", et qui ne relève pas du descriptif mais du rythmique, grâce auquel le monde se trouve "digéré " par et dans.le langage. D'autant que le paysage dont je parle, moi, a la particularité d'être désertique, dénudé, réduit à ses forces vives, primitives. C'est la phusis des Grecs, incorporée et restituée en Rythme et en Musique. Miron m'a dit un jour que j'étais peut-être l'un des plus "québécois" des poètes québécois parce que ma poésie nomme le pays physique: elle est, comme je l'ai écrit dès 1965 , bien avant que le poète Kenneth White n'en popularise l'expression dans les années quatre-vingt, une géopoétique ${ }^{1}$ du pays d'ici.

1. Yves Préfontaine, "En guise de postface" [28 juillet 1965], Les êpousailles, Parole tenue, poèmes 1954-1985, Montréal, l'Hexagone, coll. "Rétrospectives", p. 207. 
De plus, cette géopoétique baignait dans les puissances à la fois chthoniennes et célestes d'un Éros omniprésent. Au fond, je n'ai jamais écrit qu'un long poème d'amour au monde. Je bataillais avec la chair-monde. Il ne faut pas perdre de vue qu'Éros vient du chaos primitif et qu'il y a, avant lui quant à l'ordre d'importance, Dionysos, dieu de vie et de délire. Dionysos est un dieu magnifiquement dangereux, escorté de ses Ménades, les "femmes possédées". Dans mon mythe dionysiaque personnel, il y a la guerre d'amour avec et contre la Matière, à la fois sacrée et aveugle... La suite "Jusqu'au chant de matière", dans Nuaison, se termine sur ces mots: "Maintenant, les matières affluent vers ce centre de nousmêmes qui n'est plus d'humaine flamme mais de forge divine ${ }^{2}$ ". Le dieu, hélas, ne nous habite que le temps d'un mirage... Mais les Forces demeurent, indicibles, pour renouveler à l'infini, en nous, Sisyphe du Verbe, la colère de nommer.

V. et I. : La pratique de l'aphorisme ou du fragment et de la réflexion poétique sur la poésie était rare au Québec dans les années cinquante, contrairement à ce qui se passait en Europe, avec Char, Michaux et Blanchot, notamment. Qu'est-ce qui vous a poussé à l'écriture de L'antre du poème?

Y. P. : C'est sans doute pour prendre mes distances - pour parer à toute éventualité catastrophique - face aux énormes pulsions qui m'habitaient et dont certaines pouvaient être fort destructrices que j'ai très tôt pratiqué le fragment et l'aphorisme. J'ai commencé à prendre des notes sur mon sismographe personnel en même temps que prenait forme l'écriture de Boréal et des Temples effondrés, en 1954. Poésie et fragment ont été très longtemps pour moi des "supports" littéraires complémentaires. J'éprouvais le besoin d'exprimer de façon à la fois plus littérale et plus condensée mes "démons et merveilles", si je puis dire, d'ajouter à ma démarche un commentaire parallèle. Je ne connaissais pas encore René Char ni Maurice Blanchot. Ai-je été influencé par Valéry et Nietzsche, que j'avais davantage pratiqués? Je l'ignore. J'ai l'impression que cela m'est venu du dedans, comme une nécessité irrépressible, dès l'instant où j'ai commencé à mettre un début d'ordre dans le chaos qui m'habitait. Il y eut même des moments où cette forme d'expression prenait le pas sur le poème. Dans les années soixante, durant mon séjour en Europe, mais aussi dans les années soixante-dix. J'écrivais là ce qui sera le Livre de mon bord, pour reprendre le titre du négligé et admirable Pierre Reverdy. J'ai près de quarante ans de ces textes qui sont encore inédits.

V. et I. : On a souvent souligné votre précocité, et la prolixité dont vous avez fait preuve à vos débuts. On a évoqué Rimbaud, Lautréamont, dont vos poèmes d'alors avaient l'élan, l'emportement, le côté "visionnaire" aussi. On pourrait parler, pour les poètes d'ici, de Nelligan, de Jean-

2. Id., Parole tenue, op. cit., p. 401. 
Aubert Loranger, de Saint-Denys Garneau, de Claude Gauvreau et de Paul-Marie Lapointe, qui ont eu la même précocité. Pouvez-vous nous parler de vos toutes premières publications?

Y. P.: J'avais quinze ans, je crois, quand le journaliste Michel Roy, aujourd'hui sénateur, alors homme de gauche et rédacteur en chef du journal L'Autorité du peuple, présenta mes premiers textes publiés sous le titre: "Avons-nous trouvé notre Raymond Radiguet?" J'étais flatté, bien sûr, qu'on me publie à cet âge, mais un peu insulté qu'on me compare à ce jeune écrivain, prodige à sa manière, que j'avais lu mais que je considérais comme "mineur". Les textes publiés, si ma mémoire est bonne, se terminaient sur l'image d'un homme "qui s'avance dans la mer avec / au bout de ses bras coupés / un enfant mort d'être seul". Je percevais mal ce qu'il y avait là de commun avec Radiguet.

C'est à la même époque que j’ai commencé à fréquenter ceux d'entre les automatistes qui étaient encore à Montréal: Claude Gauvreau, bien sûr, mais surtout les peintres, dont les œuvres me nourrissaient à certains moments plus que la littérature. Jèn-Paul Mousseau (qui, plus tard, fera la maquette de L'antre du poème), Marcel Barbeau, Fernand Leduc (qui me fit découvrir le Divin Marquis), la magnifique Marcelle Ferron (qui illustra sur de grandes feuilles des espèces de poèmes-affiches exposées à la Place des Artistes en 1953, je crois), figurent parmi les gens que je fréquentais. J'étais fasciné par ces artistes, mais j'éprouvais en même temps, à cause de l'écart d'âge sans doute, une intense solitude. Il faut dire, toutefois, que dans ces années sombres de la "nuit québécoise", la solitude, le suicide, la déprime étaient choses largement partagées...

V. et I. : Plusieurs des écrivains précoces dont nous parlions plus haut n'ont pu atteindre leur pleine maturité, leur œuvre ayant été trop tôt interrompue par le silence, la mort ou la folie. Vous combinez, vous, la longue durée, celle d'une lente maturation, sur plus d'une quarantaine d'années, et l'instantanéité d'une éclosion poétique qui s'est imposée à vous et aura imposé votre œuvre très tôt, alors que vous n'aviez pas vingt ans. Comment, aujourd'hui, vóyez-vous l'inscription de votre ouvre dans les différentes périodes de votre vie? Y voyez-vous des moments d'urgence, où l'écriture s'est manifestée de manière plus impérieuse, et d'autres de relative accalmie, où les projets ont mûri dans le silence et le retrait?

Y. P. : Plus jeune, j'ai certes écrit sous le signe d'une urgence proprement irrépressible, pour ne pas dire panique. J'avais la forte impression d'être habité, hanté plutôt par des forces, que je confondais volontiers avec ce que je percevais des forces du monde. Et puis je crois avoir exprimé à différents moments de ma vie la hantise, l'obsession de l'espace monstrueux que nous tentons d'habiter depuis quatre siècles. Cet espace physique, chez moi et chez quelques autres, se prolonge en espace psychique, obscur, qu'il nous faut investiguer. Le "dieu du froid" auquel j'ai dédié méta- 
phoriquement le poème-titre de Boréal, c'est, au fond, le daìmon de notre nordicité. Mais contrairement à l'étymologie du terme, ce daïmon ne nous relie pas à quelque dieu. Au "néant blanc", plutôt. Ou, plus simplement, à l'acte d'être là. Comme Nietzsche, lui, désirait être "sur les cimes", proche des glaciers. Car, voyez-vous, la plupart des humains, en tout cas les euro-atlantiques, sont faits pour vivre dans un climat tempéré. Ce n'est pas tout à fait notre cas ici... L'obsession des cimes et des profondeurs abyssales de la mer ou de l'espace ne contredit pas ce besoin.

Même s'il y a continuité, thèmes fondamentaux et variations subséquentes, il est certain que mon travail obéit à des cycles de vie, à des sortes de rythmes plus ou moins excessifs. Vous connaissez le principe de "tension/détente" qui régit la plus grande partie de la production musicale occidentale. Ce principe a été brisé par la seconde École de Vienne, depuis la phase dite atonale jusqu'à la conceptualisation de la musique sérielle par Schœenberg, dans ces années fabuleuses du début du siècle. Je crois avoir inconsciemment adopté le même cheminement. Boréal, Les temples effondrés et L'antre du poème ont été conçus dans un état d'incroyable tension psychique. Cette tension atteint son paroxysme dans Les épousailles, dont j'ai tant tardé la publication pour deux raisons. La première trouve sa source dans une sorte d'effondrement que j'ai vécu, en 1958, à la fin d'un long voyage au Mexique et après mon retour de cette aventure solitaire pour moi fondamentale. Cet effondrement n'a pas duré des siècles, puisque j'ai commencé à écrire Pays sans parole au début de 1959. La seconde raison est que j'éprouvais un certain agacement face aux excès de ces textes malgré l'épuration que je pratiquais sur eux. J'étais pour moi-même un critique impitoyable: à côté de quelques textes perçus comme presque parfaits, telle "Aphrodite sortant de la mer", tant d'autres que l'on doit sculpter sans cesse pour en parfaire la tessiture!

La tension dont j'ai parlé plus haut est encore perceptible, je crois, dans Pays sans parole et Débâcle, mais la rythmique, le ton, plus "ontogénétique", si l'on me permet ce terme, changent avec À l'orée des travaux et surtout Nuaison. J'étais mûr pour d'autres voies, ou d'autres voix... Car au bout des Épousailles, un Claude Gauvreau m'attendait, avec ses hurlements proférés en dehors de toute intelligibilité... Et ses internements répétés.

V. et I.: La question du sens reste pour vous primordiale, même s'il doit s'incarner dans la matière verbale. Votre "matièrisme", comme vous l'appelez, ne vous a pas conduit à une fétichisation de la lettre, comme chez certains autres poètes, qui ont cherché à l'affranchir du sens.

Y. P.: Le lettrisme et, plus tard, le formalisme m'ont toujours été étrangers. Bien sûr, comme je le disais tantôt, la poésie est sculpture du verbe. La parole est un matériau que l'on façonne. La poésie est un "artisanat furieux", comme l'écrit René Char. Le poïen fait de toute poésie une poterie, une 
poéterie, comme dit le poète Robert Marteau. Mais ce "faire" est toujours en rapport avec un monde, un sens, sans lesquels il n'existe pas.

V. et I.: Ce "monde", vous l'avez découvert notamment à travers vos voyages. Parlez-nous de ce premier long voyage au Mexique et au Guatemala, que vous évoquiez plus haut. A-t-il apporté quelque chose à votre œuvre poétique?

Y. P.: Je suis allé au Mexique, en 1957 et 1958, avec le sentiment de découvrir mon américanité. Je n'avais pas voyagé hors du pays avant cette longue aventure solitaire. Ce premier voyage a été un choix, sachant que plus tard j'irais en France et en Europe. C'est peut-être sous la double influence de Kerouac (j'avais lu On the Road, entre autres) et d'Artaud (ses écrits mexicains) que je suis parti seul, mon baluchon sur l'épaule, vers le Sud. J'ai écrit là-bas quelques-uns des poèmes des Épousailles et un certain nombre d'aphorismes et de fragments. Mon univers baroque de l'époque s'accordait bien avec l'imaginaire mexicain.

Je peux dire que le Mexique m'a apporté des images, dés sédiments de sensations, par sa profondeur historique formidable, qui remonte aux civilisations méso-américaines, que je connaissais par mes lectures des travaux de Jacques Soustelles et d'autres. Avant même de faire des études d'anthropologie, je m'intéressais de près à ces cultures, entres autres au Popol-Vub (le Livre des conseils), et aux commentaires qu'on a faits.

Le Mexique a drainé une bonne part de mon imaginaire. Après mon retour j'aurais aimé, dans la suite de Boréal, écrire quelque chose de "tropical", notamment en souvenir de mon séjour au Yucatán et au Chiapas puis au Guatemala, où je n'ai pu rester longtemps à cause du climat de guerre civile qui y régnait déjà, après que la C.I.A. et ses généraux indigènes de service eurent endigué, peu de temps auparavant, l'un des premiers gouvernements de gauche démocratiquement élu en Amérique latine. L'atmosphère militaro-fasciste y était irrespirable, mais ne m'a pas empêché, pour une brève période, de visiter les fameuses ruines mayas, dont cèrtaines venaient à peine d'être dégagées. Ces villes anciennes d'une immense civilisation n'ont pas cessé de me fasciner: elles sont nées $i c i$, dans l'ici américain, et font partie de nous, aussi.

V. et I. : On a l'impression que ce continent jeune, nouveau, qu'est l'Amérique, vous le percevez à travers ce qu'il a de plus "ancien", soit les civilisations précolombiennes aujourd'hui disparues, alors que la vieille Europe, vers laquelle vous tourne votre culture littéraire, ne vous intéresse qu'à travers ses productions les plus "modernes", depuis les surréalistes jusqu'à nos jours.

Y. P.: J'ai aussi été, très tôt, fasciné par le jazz et la littérature noire américaine, qui sont l'expression "moderne" de l'américanité. J'ai notamment traduit, pour la radio, plusieurs poèmes de Langston Hughes et d'autres, que je lisais dans les émissions de jazz que j'animais: J'ai aussi traduit des 
poèmes mis en chanson par Abbey Lincoln, qui avait une voix magnifique, et le batteur Max Roach, son compagnon de l'époque... J'ai été l'un des premiers à faire un cours sur les littératures noires d'expression française lors de mon passage comme professeur invité à l'Université Laval. L'afro-américanité, comme l'amérindianité, m'aura donc fortement marqué depuis fort longtemps. Je n'ai jamais vu de contradiction entre le fait d'écouter Coltrane, Davis et Monk, ou encore la musique balinaise, et le fait d'écouter Bach ou Webern.

V. et I.: Si l'on revient maintenant à votre parcours, on remarque une chose assez étonnante. La réunion et la publication de vos poèmes en recueil suivent parfois de plusieurs années le moment de leur écriture: Débâcle a paru en 1970, mais regroupe des poèmes écrits entre 1960 et 1963; il faudra ensuite attendre 11 ans pour lire Nuaison, paru en 1981, bien que les poèmes qui lè composent furent écrits dans la continuité de Débâcle, de 1964 à 1970; et il faudra patienter encore 6 ans pour que paraisse Le désert maintenant, en 1987, où nous pouvons lire des poèmes écrits sur près de 15 ans, de 1971 à 1985; enfin, nous attendons encore la parution de Devenir, il n'est rien d'autre (l'ex-Verbe Etre), dont quelques extraits ont été publiés en revue depuis maintenant une dizaine d'années. Retravaillez-vous beaucoup vos poèmes et leur organisation en livre avant d'envisager leur publication?

Y. P. : J'avoue souffrir d'une certaine procrastination. C'est une maladie terrible! Une peste mentale! Mais ne serais-je pas rongé par cette plaie, cela ne changerait rien à l'attitude circonspecte qui est la mienne face à l'acte de publier. À vingt ans, sur ce plan, je suis "parti en peur". Puis il y eut une sorte de rupture. Publier deux livres la même année, en 1957, je crois que cela ne s'était jamais vu auparavant au Québec. De la fin des années cinquante à la fin des années soixante, j'ai été plutôt gâté par la critique. Et mon métier d'homme de radio de même que mon engagement politique prolongeaient une certaine image, je suppose. Mais il s'en trouve toujours pour vous faire payer cher votre précocité, si précocité il y a.

À cela s'ajoutent des considérations plus concrètes. Dans les années soixante, j'ai été rédacteur en chef de la revue Liberté, dont mon ami Hubert Aquin était le directeur. Simultanément, en 1962, j'étais retourné à l'Université de Montréal pour y entreprendre un B. Sc. et une maîtrise en anthropologie. J'avais aussi mon travail à Radio-Canada. Je n'avais même pas le temps de m'occuper du R.I.N., dont je fus l'un des membres fondateurs, soucieux que j'étais pourtant que la gauche du mouvement ne perde pas le terrain qu'elle occupait. En 1965, je suis parti six ou sept mois aux Antilles françaises pour y glaner les données de ma thèse. L'année suivante, je me mariais et partais pour un séjour de quatre ans à Paris afin d'y poursuivre des études de doctorat en sociologie de la culture. Si ma mémoire est bonne, j'avais donné le manuscrit de Pays sans parole à Gaston Miron avant mon départ pour les Antilles. Il devait le 
publier quelques mois plus tard, mais le livre n'a finalement paru qu'à l'automne 1967.

Mon silence des années soixante-dix repose sur de tout autres raisons. Je suis revenu d'Europe en août 1970, notamment pour occuper un poste de professeur à l'Université McGill. À peine le temps de préparer mes cours que la Crise d'Octobre nous tombe dessus! J'avais vécu Mai 68 à Paris, deux ans auparavant. En août de la même année j'étais à Prague, que j'ai dû quitter quarante-huit heures avant l'invasion soviétique, pour revenir par la Yougoslavie. On n'a pas chômé durant ces années là! Ici, Débâcle suivi d'A l'orée des travaux est mis en librairie, en octobre 1970, sans lancement, bien entendu, étant donné les circonstances: Miron est en prison. Les flics et un militaire me rendent une visite de politesse... Je suis sur leur liste. On ne m'arrête pas parce que; dit l'un d'eux, je ne suis pas dangereux: je travaille pour les anglais! Insulte suprême, mais qui m'aura évité les horreurs qu'ont vécues tant de collègues et amis. J'étais revenu fort déprimé de Paris. Je ne sais pas comment j'aurais survécu aux Ordres...

Puis il y eut les premières critiques du livre. Dans La Presse, Martel le juge "dépassé". Débâcle aurait peut-être pu paraître dans Pays sans parole, qu'il prolonge en effet, mais le critique n'a sans doute pas pris la peine de lire $\bar{A}$ l'orée des travaux, qui rompt radicalement avec les recueils précédents. Dans Le Devoir, un certain Pilon, autrefois collègue sinon ami, éructe non pas une critique, même négative, mais un règlement de compte. On ne fonde pas une critique sur l'animosité personnelle, quelles qu'en soient les raisons. À la limite, on fait silence. Mais tel n'était pas le fonctionnement de notre "système littéraire". Axel Maugey répondra au susdit Pilon en janvier 1971, dans le même journal, mais le mal était fait.

Deux mois plus tard, au printemps, eut lieu l'horrible congrès des écrivains québécois, où ceux-ci, au lieu de se "serrer les coudes" devant l'assaut innommable que nos gouvernements avaient lancé contre la collectivité, s'entre-déchirèrent allègrement. J'ai été parfaitement écœuré, blessé, par les critiques acerbes et malhonnêtes entendues lors de cette rencontre, même de la part de prétendus "amis"... Ce sont là quelquesunes des raisons qui expliquent mon silence des années soixante-dix.

V. et I. : Comment résumeriez-vous les grandes lignes de votre parcours, en comparaison, notamment, avec la façon dont la critique a vu évoluer votre œuvre d'un lyrisme foisonnant, attaché à une vision cosmique et mythique du langage et du monde; vers une plus grande ascèse prosodique et rhétorique de même qu'un resserrement thématique sur des problèmes de nature plus existentielle ou personnelle, empreints d'une certaine spiritualité ? Voyez-vous des périodes, des ruptures ou des continuités, dans votre travail poétique depuis ses débuts? 
Y. P.: Continuité dans la rupture, dirait un politicien... Il y eut d'abord cette phase que je qualifierais, avec réserve, de "mystagogique", d'initiation à des mystères qui n'étaient peut-être mystérieux que pour moi... C'est ce que je nomme, dans le premier "Indice" de ma rétrospective, une "mystique spatio-cosmique radicale", un "matérialisme phonique excessif ", qui n'en mène pas moins, peu à peu, à l'enracinement plus évident de Pays sans parole, à la graduelle simplification des écritures qui vont suivre.

Mais simplification n'est pas dénuement francișcain, tant s'en faut. Il n'y a pas eu mutation brutale, à cette époque, du style et des thèmes, mais glissement vers une clarté, un éclaircissement progressif d'une matière verbale et sémantique jusque-là obscure. Ma propre "fondation du territoire", pour reprendre le très beau titre d'un essai de notre ami Paul Chamberland, était déjà inscrite en filigrane dans Boréal et L'antre du poème. Quand je parle, dans ce dernier livre, d'une "matière de pensée" que "l'ouvrier de l'étape ultime transmue en concrétions de hautes paroles issues de contrée", c'est de cet immense pays à peu près innommé qu'il s'agit, de ces vastes étendues où même les autochtones les plus nomades ne mettaient pas les pieds. Songez que certains groupes amérindiens et inuits considéraient les régions centrales du Nouveau-Québec comme une sorte d'Enfer... Ce sont là, sans doute, les bourgeons de la poésie dite du pays...

Mais laissez-moi dire ici, une fois pour toutes, l'irritation que j'ai toujours éprouvée d'être identifié à cette "poésie du pays". Je préférerais qu'on dise, lisant mes livres: voici un homme qui a vécu et vit encore dans un pays menacé; cet homme est obsédé par les limites du langage; cet homme s'est perçu, comme d'autres avant lui, tel un "rôdeur des confins" psychiques; il en a payé le prix à quelques reprises; il porte en lui l'énormité de son pays physique, sa géologie, ses plantes, ses pierres, son fleuve, son Grand Nord, son climat démentiel; il se heurte au mystère de l'être; il se heurte à la très vraisemblable inexistence d'un dieu tutélaire, au silence, à l'abyssale absurdité du comportement des hommes et, très honnêtement, de son propre comportement; cet homme crie, il est seul, il est avec d'autres, il rit mais le plus souvent il pleure; il pleure, comme tout un chacun, en écoutant les nouvelles à la télé; il pleure sur lui, sur ses amis qui commencent à disparaître, sur sa compagne aussi, parce que rien n'est donné, rien n'est facile; cet homme, après s'être pris pour un démiurge verbophore, aura tenté de traduire les grands et les petits séismes qui agitent le monde et l'agitent...

V. et I.: Verbophore, mystagogue... On pense à votre fascination pour les mythologies. Vos tout premiers recueils recèlent un ton épique mythique, au sens anthropologique du terme - qui les apparente aux grands récits de fondation, aux cosmogonies et aux genèses, aux mythes d'origine: ils semblent viser le recommencement du monde, que le 
poème re-nomme, au plus près de ses sources, là où la chair devient verbe, là où la chose vient au mot. Vous écrivez dans Les épousailles (1956-1957): "Épeler la source... tel est le dire de matière ${ }^{3}$ " puis, en 1988, dans l' Indice" qui ouvre votre rétrospective: "C'était au temps où les mots fusaient à la source même du volcan ${ }^{4}$." On pense aux philosophes présocratiques, à la Théogonie d'Hésiode, au De natura rerum de Lucrèce, à tout le bagage de mythes qui vous étaient familiers, par l'intérêt que vous portiez à l'anthropologie. Pouvez-vous nous parler de cette fascination pour l'origine, pour la gestation et la genèse communes de la chair et du verbe?

Y. P.: Lucrèce, dites-vous? Je me souviens de mon père me le traduisant directement du latin au français quand j'avais treize ou quatorze ans... Je ne me souviens pas d'un temps où je n'ai pas éprouvé une obsession des Origines. J'ai l'impression d'être né avec les yeux fixés sur le Big Bang (dont nous ne connaissions rien à l'époque) et sur l'éternelle question: qu'est-ce qu'il y a derrière ce foutu bordẹl? Une cause première? Un dieu anonyme que nous cherchons comme le téton de maman et à qui les hommes ont donné tous les noms imaginables, à propos duquel ils ont pensé les concepts les plus raffinés et imaginé les formes les plus incongrues et les plus cruelles? Une "incongruité monumentale", en effet, selon la belle image d'André Pieyre de Mandiargues, ce dieu de silence absolu!

Permettez-moi de vous citer un passage d'un texte intitulé "La métaphorisation des origines: une quête limite", écrit il y a quelques années, où je répondais précisément à votre question: "Le postulat premier à l'époque, [...] c'est que l'art procède d'un rapport de forces entre le monde perçu comme une menace quasi guerrière et un moi qui s'affirme contre lui. [...] La Nature omniprésente est ressentie comme un énorme bruit, un vacarme aveugle et chaotique qu'il faut contrôler par un Verbe ordonnateur et forcené (le gouffre qui fascine, le vertige qui méduse mais qu'il faut maîtriser sous peine de mort); à ce vacarme, il ne peut y avoir qu'une réponse à sa mesure, une nomination bruyante; il faut tenter d'organiser ce bruit par un autre bruit tout aussi puissant. Il faut ajouter à ces perceptions extrêmes l'influence évidente de la dimension physique de notre pays-continent ${ }^{5}$."

V. et I.: Est-ce que la lecture de la Genèse et des textes bibliques a eu une grande influence sur vous à cette époque?

Y. P.: La Bible est un fort beau livre, un document unique, mais pas plus, à mes yeux, que le Bhagavad-Gîtâ de l'Inde, le Popol-Vub ou le Chilam

3. Ibid., p. 157.

4. Ibid., p. 9.

5. Id., "La métaphorisation des origines: une quête limite", Joseph Melançon (dir.), Les métaphores de la culture, Québec. CEFAN, Presses de l'Université Laval, 1992, p. 214. 
Balam, ces équivalents mayas de nos textes sacrés, étrangement plus proches de la réalité scientifique de la "création". Il y est question de longs cycles où le dieu créateur rate son coup en créant l'espèce humaine, s'y prend à plusieurs reprises pour parfaire son travail tout au long de ces cycles... C'est fort loin de cette formidable idiotie de la création du monde en six jours et le septième jour, Dieu se reposa. S'il avait déjà besoin de repos, il aurait dû nous foutre la paix: nous aussi on se reposerait, pour l'éternité...

Une seule pensée de Lao-Tseu m'apporte davantage que tous les Ecclésiastes réunis. Outre les culpabilités dévorantes, les tabous sexuels et tous les fantômes qui hantent l'Ancien Testament, ce que j'ai retenu de ce monde de géhenne et de veuves lapidées, c'est la notion de charité... La compassion, toutefois, était présente chez Siddharta Gautama, le Bouddha, l'Éveillé, près de six siècles avant Jésus-Christ.: Encore une fois, plus que dans les textes bibliques, j'ai trouvé dans les fragments foudroyants qui nous sont parvenus des présocratiques, par exemple, d'Héraclite surtout, de quoi nourrir mon appétit d'une connaissance qui ne sépare pas le poétique du philosophique, ni l'éthique de l'esthétique.

V. et I. : L'écriture, chez vous, a toujours été liée à la connaissance, à une forme du "co-naître" au sens claudellien ou à une gnoséologie au sens ancien.

Y. P.: En effet, je ne crois pas en une poésie qui ne contribue pas à ce qu'on peut appeler l'ontogenèse, non pas au sens biologique de la maturation d'un être depuis sa cellule jusqu'à sa forme génétiquement déterminée (ce qui est proprement le "naitre"), mais dans le sens plus philosophique d'une co-naissance de l'être au monde et aux autres par et dans le langage, y compris par et dans la parole poétique. Je ne crois pas en une littérature, roman ou poésie, qui n'agrandit pas la perception et la conscience que j'ai du monde et des êtres, qui ne les fait pas croître et mûrir. La poésie, en ce sens, est un mode d'accroissement de nos perceptions, en même temps qu'elle est, dans l'acception chimique de l'expression, un "précipité de sens", dont les aphorismes et les brefs poèmes d'un René Char, par exemple, sont l'une des plus belles illustrations, et dont l'écriture fragmentaire d'un Nietzsche, à travers lequel j'ai découvert les présocratiques, peut être considérée comme l'un des exemples les plus forts.

V. et I. : On considère souvent votre poésie comme dionysiaque, secouée par l'Hybris, si cher à Nietzsche. Une sorte d'épicurisme du langage traverse votre poétique, tendue entre deux pôles: un désir effréné de connaître, de "faire jouir la pensée", dirait Ponge, et un désordre réfléchi de tous les sens, comme le prescrit Rimbaud.

Y. P.: Je partage aussi la colère de Nietzsche contre la culpabilité léguée par le judéo-christianisme. Je me bats encore contre cet héritage, qui a étouffé longtemps toute forme de jouissance, y compris poétique. 
V. et I. : N'y a-t-il pas, pourtant, dans l'usage que vous faites du verset ou de la laisse et d'une prosodie qui emprunte à la psalmodie quelque chose que vous retenez, sans doute plus dans la forme que dans l'idée, des grands textes qui ont fondé le christianisme?

Y. P.: Il y a bien sûr de grands textes, comme l'Apocalypse et le Cantique des cantiques, de même que certains prophètes; je songe aux visions troublantes, hallucinées, du livre d'Ézéchiel, notamment. Mais, quand je parle de l'héritage judéo-chrétien, c'est au sentiment de culpabilité, aux tabous, sexuels et autres, qu'il nous a laissés que je pense, toutes choses qu'on ne retrouve pas dans le bouddhisme ni dans les religions amérindiennes, par exemple. Celles-ci concernent davantage une espèce de force cosmique qui n'a pas de nom, innommée parce que non individuée. On peut penser au panthéisme d'un Spinoza, si l'on veut avoir un point de comparaison dans la pensée occidentale... Cela dit, je ne rejette pas toute spiritualité chrétienne, notamment lorsqu'elle prend une forme poétique comme chez Jean de la Croix, Thérèse d'Avila et Sor Juana Inés de la Cruz, qui sont des aérolithes à l'intérieur d'un système plutôt monolithique... À la limite, je peux être fasciné par le comportement d'un saint François d'Assise, que je trouve remarquable. D'ailleurs, dans mes fragments inédits; il y a quelques textes sur la tentation monastique et sur l'aspect idéalement "communiste" de certaines communautés... Mon rejet porte en fait sur toutes formes de religions monolithiques, supposément révélées, qui oublient le sens étymologique du verbe religare, lequel désigne le fait de "relier" ou "rassembler" ce qui reste distinct et différent, non pas de tout "fondre" dans une même "unité ", une même domination des "âmes" sous la férule de dogmes aussi absurdes les uns que les autres.

V. et I.: Une "religion" du verbe, sans Dieu?

Y. P.: Il y a une phrase extraordinaire, tirée des textes védiques indiens et citée, je crois, par Maeterlinck, qui dit ceci : "Dieu ignore ce 'qu'il est et l'homme ne saura jamais rien". Voilà qui est d'un optimisme radieux, n'est-ce pas? Outre les Veda et les Upanishad, il y aussi la Kabbale, dans la tradition judaïque, qui m'a longtemps intéressé, par sa conception de la création selon trente-deux voies, correspondant aux vingt-deux lettres de l'alphabet hébraïque et aux dix "sephirot " qui sont des nombres ou des "rayonnements". C'est un beau mythe, comme d'autres, tout aussi beaux, dont on a moins parlé.

V. et I. : Certains pourraient dire, néanmoins, que le titre que vous comptiez donner à votre prochain livre de poésie, Le verbe Être, possède d'évidentes connotations chrétiennes, à moins qu'il ne renvoie à une tradition néoplatonicienne, alors que le nouveau titre, Devenir, il n'est rien d'autre, semble plus héraclitéen?

Y. P. : Je parle trop de mes titres! En effet, les responsables des Éditions de l'Hexagone ont donné le titre annoncé de mon prochain livre, Le verbe 
Etre, à un recueil de Paul Chanel Malenfant qui, de fait, n'est aucunement responsable de cet incident malencontreux. On trouvait que le titre original de son livre ressemblait trop à celui d'un recueil de François Charron. On lui a donc proposé le mien, alors que j'avais bien avant, dès 1991, publié plusieurs extraits de mon propre livre dans Possibles, Conjonctures, Estuaire, Ritmica et d'autres revues, toujours avec la mention "Le verbe Etre, extraits d'un livre à paraître ".

Mais revenons à votre question. La notion de logos, chez les Grecs, est plus vieille et plus fondamentale que celle du verbum latin. Le "verbe fait chair", auquel vous faites référence, m'intéresse d'un point de vue anthropologique parce qu'il appartient encore aux mythes nourriciers. C'est toutefois un mythe qu'il faut réinventer, comme dans toute mythologie, et réinventer par la poésie. En fait, je m'intéresse au "principe", qui est aussi l'objet des religions. Ce principe, je l'appelle cela, comme dans les Upanishads. Tout simplement. Et non pas Theos, Dieu, ou autrement. Le cela, c'est le principe premier. Peut-être... En dehors du scepticisme et de l'agnosticisme absolus, tout choix de réponses aux questions essentielles relève d'un acte de foi, qu'il penche vers les divers matérialismes ou vers la gamme des spiritualismes multiples.

V. et I. : Ne peut-on pas voir dans un poème comme "Non-lieu ${ }^{6}$ " - qui sonne étrangement comme Non-dieu - l'expression de ce pur cela, d'un lieu vidé de lui-mème et de toute substance, en éternel devenir, comme tout espace qu'on désigne déictiquement, en pointant du doigt vers les différents points de l'étendue, disant ou écrivant "nous qui sommes d'ailleurs tout en étant là, nous perdant là, etc."? Le cela ou le là du verbe, qu'on retrouve aussi dans votre prochain livre, n'est-ce pas une façon de se libérer non seulement du poids de Dieu mais de la gravité et de la pesanteur du sol, de la terre, de toute cette matière en fusion qui pèse sur la langue de vos premiers poèmes, tout en lui donnant son leste, sa plénitude et ses assises?

Y. P.: Oui, je suis sans doute au-delà. Dans une "Gravitation vaguement zen ", comme dit le titre d'un des derniers poèmes du Désert maintenant ${ }^{7}$. Mais je ne renie aucunement mes premiers textes, où prend racine le faisceau de thèmes que je n'ai cessé de traiter depuis et qui, avec le temps, se sont sans doute décantés. Il n'y a pas de contradiction, rien qu'un déplacement, entre le fait que je suis présentement plongé dans une quête qu'on peut bien appeler spirituelle, puisqu'elle relève du religare, du "rassembler", dans une forme et un rythme épurés, et le fait que j'ai vécu, plus jeune, la rutilance excessive, le rougeoiement permanent, le fer rouge qui me traversait chaque fois que je me mettais à écrire...

6. Voir Le désert maintenant, Parole tenue, op. cit., p. 481-483.

7. Ibid., p. 490. 
V. et I.: Ne peut-on pas dire que la figure emblématique des premiers livres est le cri, alors que celle des derniers serait le silence?

Y. P.: Ou le murmure. Qui paraît à l'opposé des Épousailles. Mais le lyrisme qui m'habitait et m'habite encore revient, subrepticement, à la fin du prochain livre, qui s'achève sur une longue incantation où je retrouve le goût de ce que j'appelais autrefois une pangraphie, c'est-à-dire une volonté de tout dire, de tout écrire. Ce poème s'intitule "Habitez-moi "...

V. et I. : Après avoir dit, dans les premiers textes, habitons le monde, le pays, voilà qu'aujourd'hui vous renversez ce rapport, en demandant au monde de vous habiter... Le paysage se trouve tout entier ingéré et digéré en inscape, en intériorité, comme vous le disiez plus tôt. Est-ce une façon d'affranchir l'acte poétique, tout entier intériorisé, de l'action politique à laquelle il a d'abord donné lieu?

Y. P. : J'appartiens à une génération qui n'a pas eu le choix : il fallait agir. Si j'étais né dans un pays qui possède une profondeur historique, comme la France, l'Angleterre, l'Italie ou l'Allemagne, j'aurais certes été engagé sur un plan social mais je ne me serais sans doute pas autant investi, comme je l'ai fait à certains moments de ma vie, dans des actions politiques qui dévorent énormément de temps et d'énergie, qu'on ne peut, dès lors, consacrer à l'écriture. Plusieurs poètes de ma génération auraient eu une œuvre plus considérable si le pays ne risquait pas à tout moment de basculer dans un no man's land culturel. Dans un article de Québec libre, publié en 1959, je parlais déjà de la "louisianisation" éventuelle du Québec: j'avais créé ce néologisme, qui est resté par la suite. Notre situation géopolitique nous oblige à l'engagement. Il y a donc, pour moi, une continuité entre l'acte poétique et l'action politique, même si je n'ai pas été aussi présent sur cette scène qu'un Gérald Godin, par exemple.

J'ai tout de même été directeur de cabinet du $\mathrm{D}^{\mathrm{r}}$ Camille Laurin, alors ministre d'État au Développement culturel. Un directeur maladroit, sans doute, mais au moins j'aurai été fidèle à moi-même et aurai accompli ce que je considérais comme un devoir élémentaire. Pour m'être éloigné du milieu littéraire, je n'ai pas pour autant abandonné ce que j'appelle depuis des lustres mon "état de québécité". Le cabinet politique, cependant, n'est pas un "job" de poète, je l'ai souvent dit... J'en suis sorti avec un ulcère d'estomac au bord de la perforation! Mais je ne suis pas peu fier d'avoir participé avec mes petits moyens à l'extraordinaire et dévorante dynamique de ces annéeslà : l'application de la loi 101, de la Charte de la langue française, la première politique du droit d'auteur, la première politique scientifique québécoise, le livre blanc sur le développement culturel (malheureusement sabordé plus ou moins), etc. Mais l'échec du premier référendum fut pour moi un coup de massue sur le crâne... Je ne parle pas du deuxième...

V. et I. : Depuis quelques années vous semblez vivre une sorte de double retraite du monde littéraire et du monde politique dans la mesure où vos 
publications et vos prises de position publiques se font plus rares. $\mathrm{Ne}$ peut-on parler, dans votre cas, d'un passage du politique à l'éthique ou au poéthique, la poésie étant le lieu d'interrogation radicale des "valeurs"?

Y. P.: Les préoccupations éthiques ont toujours été là. Elles sont sans doute plus manifestes aujourd'hui. Elles se sont accentuées depuis sept ou huit ans. L'éthique est pour moi la quête d'un plus être. Je ne sais pas précisément où je vais. Je vais comme ça vient, on n'a pas le choix. Mais ce que j'écris est de plus en plus silencieux, de plus en plus aérien, y compris par le rythme, qui n'a plus rien à voir avec le culte du heurt, du choc, qu'on trouve au début. C'est plus flottant: une manière d'état d'apesanteur. J'aspire par là, sans doute, à une sorte de sainteté laïque... 